\title{
Disintegration Measurement of Sugar Coated Tablets by Thermal Analysis
}

\author{
Yoshinobu Nakai, Shinichiro Nakajima, ${ }^{1)}$ and Hidenori Kakizawa ${ }^{1 \alpha)}$ \\ Faculty of Pharmaceutical Sciences, University of Chiba.1)
}

(Received May 22, 1974)

\begin{abstract}
The disintegration process of sugar coated tablets was examined in the acidic and alkaline test solution by thermal analysis. The thermogram revealed the disintegration in detail, and clearly showed the disintegration process corresponding to the component of the coating layer; the dissolution of the fine sugar particles contained in the sugar layer, the reaction of calcium carbonate (dusting powder) with hydrochloric acid, the inhibition of the medicinal release by the water protective film, the medicinal release from the core tablet, and the reaction of ascorbic acid contained in the core tablet with heavy metal ions in the test solution. The sugar coated tablet without the water protective film was compared to the tablet with the film, and influence of the film on the medicinal release was examined. Ageing effect of the film on the dissolution was also examined from the thermograms of the 18 months stored tablets. It was found that the delay of dissolution was caused by the chemical and/or physicochemical change of the film properties.
\end{abstract}

Medicinals releasing from a tablet pass through two processes: tablet disintegration and dissolution of the dispersed particles. The processes are affected by many factors including tablet structure, particle size and rate of solution. General methods which have been used for disintegration measurement cannot elucidate the disintegration and dissolution process in detail. The processes of an uncoated tablet have been analyzed by thermal method reported in previous papers. ${ }^{2)}$ Disintegration of a coated tablet includes an additional factors to that of uncoated tablet. The coating layer must be dissolved prior to disintegration of the core tablet, and release rate of the medicinals in the tablet is affected by the properties of the layer, for example, thickness and uniformity of water protective coating film. Manufacturing condition and shelf life would affect the coating layer and result the protraction of medicinal release from the tablet.

In this report, disintegration and dissolution process of sugar coated tablets were examined in detail by thermal analysis. The inhibiting effect of a protective coating film on the medicinal release and the effect of storage on the inhibition were also examined.

\section{Experimental}

Calorimeter- - The twin type calorimeter reported in previous paper ${ }^{3)}$ was used. The calorimeter cells of Dewar's vessels which had the same structural and thermal characteristics were fixed in the calorimeter assembly. Temperature difference between two cells was detected by thermisters as one of the cells was used for reference. Temperature and differential temperature with time were recorded at the same time. The accuracy of temperature measurement was $2 \times 10^{-3}{ }^{\circ}$, and the heat capacity of the cell containing $200 \mathrm{ml}$ test solution was about 240 calories. On each running of the disintegration measurement, exact value of the heat capacity and cooling constant were measured. Thermoregulated water bath surrounding the calorimeter cells was kept at $37 \pm 0.01^{\circ}$. Test solution contained in the cells was kept stirring at $300 \mathrm{rpm}$ during the thermal measurement. Measurement of tablet disintegration started after the steady state

1) Location: 1-33 Yayoi-cho, Chiba; a) Present address: Product Development Laboratories, Sankyo Ltd., 1-2-58 Hiro-machi, Shinagawa-ku, Tokyo.

2) a) H. Nogami, J. Hasegawa, and Y. Nakai, Chem. Pharm. Bull. (Tokyo), 7, 331, 337 (1959); b) Y. Nakai, Y. Kubo, ibid., 8, $634(1960)$; c) Y. Nakai, ibid., 8, $641(1960) ; 9,796(1961)$; d) H. Fukuzawa, Y. Nakai, K. Shizu, and S. Nakajima, Yakuzaigaku, 30, 21 (1970).

3) H. Fukuzawa, Y. Nakai, K. Shizu, and S. Nakajima, Yakuzaigaku, 30, 21 (1970). 
TABLE T. Component of Sugar Coated Tablets mg/Tablet

\begin{tabular}{llrr}
\hline & & Tablet A & Tablet B \\
\hline \multirow{2}{*}{ Core tablet $\left.{ }^{\prime}\right)$} & thiamine hydrochloride & 13.48 & 13.48 \\
& riboflavin & 5.25 & 5.25 \\
& pyridoxine hydrochloride & 5.25 & 5.25 \\
& ascorbic acid & 162.00 & 162.00 \\
& nicotinamide & 52.50 & 52.50 \\
& wheat starch & 40.52 & 40.52 \\
& talc & 3.00 & 3.00 \\
Water protective film & magnesium stearate & 3.00 & 3.00 \\
& cellulose acetate phthalate & 1.00 & - \\
& acetylmonoglyceride & 0.50 & - \\
& sucrose & 230.00 & 230.00 \\
& calcium carbonate & 112.00 & 112.00 \\
& talc & 69.00 & 69.00 \\
& gelatine & 1.60 & 3.60 \\
\hline
\end{tabular}

a) diameter of core tablet: $8 \mathrm{~mm}$

of temperature distribution in the cells was recognized. The state could be ascertained from the base line on the recorder.

Sugar Coated Tablet_- Two kinds of sugar coated multivitamine tablet, A and B, were manufactured. Table I lists the components of tablet $\mathbf{A}$ and $\mathbf{B}$. Core tablets of one lot were divided into two parts and each part was used for coating of $\mathbf{A}$ and $\mathbf{B}$. Both coated tablets had same component parts except for cellulose acetate phthalate (CAP) and acetylmonoglyceride in the coating layer of tablet $\mathbf{A}$. Tablet $\mathbf{A}$ was coated by a thin film of CAP before the coating of sugar layer while tablet $\mathbf{B}$ was directly coated by sugar layer in the absence of the CAP film. CAP was used for the material of water protective film in this experiment. Shellac was not used for the film because of uncertainty of its chemical and physicochemical properties. The disintegration time by JP VII method was 38 minutes and 18 minutes for tablet $\mathbf{A}$ and $\mathbf{B}$ respectively in the acidic test solution, and that in the alkaline test solution was 30 minutes and 17 minutes for $\mathbf{A}$ and $\mathbf{B}$. 50000 tablets were coated for each kind of the tablets by the manual sugar coating procedure.

Test Solution-Acidic test solution was pH 1.2 buffer solution of dilute hydrochloric acid $(10 \%)$ $24 \mathrm{ml}$ and sodium chloride $2 \mathrm{~g}$ in $1000 \mathrm{ml}$ of distilled water. Alkaline test solution was $\mathrm{pH} 8.3$ solution of sodium bicarbonate $15 \mathrm{~g}$ in $1000 \mathrm{ml}$ of distilled water.

Analytical Method of Riboflavin_-Riboflavin releasing from a tablet was measured by fluorometric analysis. Fluorometer, Hitachi MPF-2A, was used, and the excitation and emission spectra were 360 and $510 \mathrm{~nm}$ respectively.

\section{Result and Discussion}

\section{Disintegration of Tablet $\mathbf{A}$ in the Acidic Test Solution}

Fig. 1 shows the disintegration thermogram of tablet $\mathbf{A}$ in the acidic solution in terms of temperature (solid line) and differential temperature (dotted line) as a function of time. The thermogram shows the successive and complicated changes in temperature and differential temperature corresponding to the properties of the coated tablet. Each change of the thermogram was interpreted in terms of the component and the structure of the tablet as follows;

Interval $\mathbf{a}-\mathbf{b}$ Temperature fell immediately after the tablet dropped into the test solution. This endothermic change is due to the dissolution heat of the fine sugar particles coating the tablet surface.

Interval $\mathbf{b}-\mathbf{c}$ Temperature highly rose from $\mathrm{b}$ to $\mathrm{c}$. This exothermic change is due to the chemical reaction of calcium carbonate in the acidic solution. The exothermic reaction of calcium carbonate in acidic solution has been examined in the previous paper. ${ }^{4}$ Calcium carbonate was used for dusting powder in the coating and contained in an inner part of the coating layer.

4) H. Nogami, J. Hasegawa, and Y. Nakai, Chem. Pharm. Bull. (Tokyo), 7, 331 (1959). 
change was due to the heat diffusing from the outer part of the calorimeter through the cell wall since the rate of the temperature change accorded with the cooling constant of the calorimeter. These results indicate that ascorbic acid decomposed exothermically in presence of a trace of heavy metal ions in the solution. It follows that the exothermic temperature change in the period from e to $f$ in Fig. 1 was due to the chemical reaction of ascorbic acid contained in the core tablet. Differential temperature curve in Fig. 1 shows the maximum rate of the medicinal release from the tablet occured at time $t_{3}$.

\section{Disintegration of Tablet B in the Acidic Test Solution}

The thermogram of tablet $\mathbf{B}$ without protective coating film was similar to that of tablet A except the point $c$ couldn't be distinguished from point $d$ as shown in Fig. 4 . It can be easily shown that the dissolution of the core tablet occurred immediately after the reaction of calcium carbonate. The core tablet began to dissolve at time $t_{\mathrm{c}}\left(=t_{\mathrm{d}}\right)$ and the dissolution of the core tablet ended at $t_{e}$. The maximum rate of the dissolution was found at $t_{3}$ on the thermogram. Comparison of Fig. 1 and Fig. 4 indicates that tablet B disintegrated and dissolved more rapidly than tablet $\mathbf{A}$.

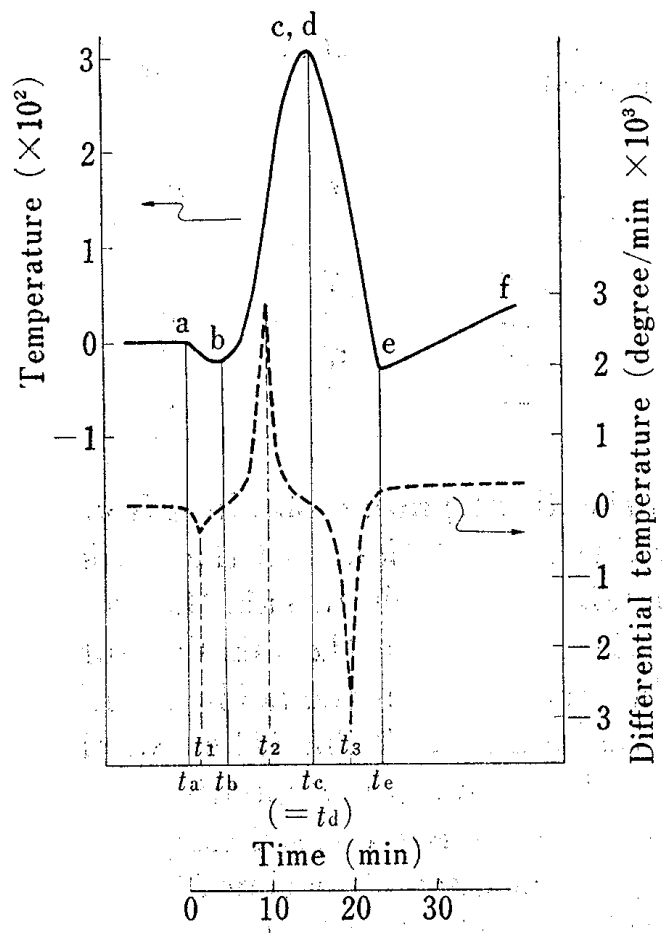

Fig. 4. Disintegration Thermogram of Tablet B in the Acidic Test Solution

$\longrightarrow$ : temperature

: differential temperature

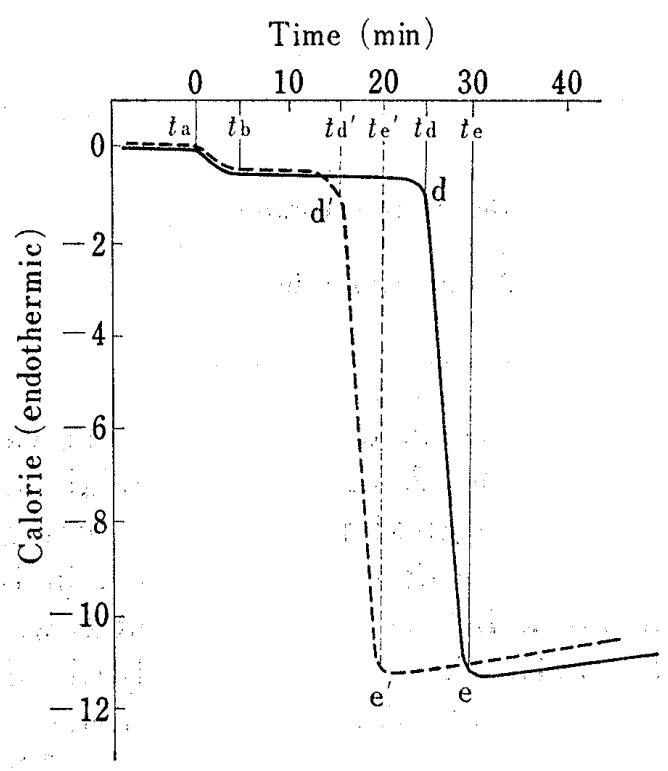

Fig. 5. Thermograms of Tablet $\mathbf{A}$ and $B$ in the Alkaline Solution

- $:$ tablet $\mathbf{A}$

\section{Disintegration of Tablet $A$ and $B$ in the Alkaline Test Solution}

Fig. 5 shows the thermograms of disintegration of tablet $\mathbf{A}$ and $\mathbf{B}$ in the alkaline solution in terms of calorie as a function of time. The sugar layer of the tablets dissolved in the period between $t_{\mathrm{a}}$ and $t_{\mathrm{b}}$ in the same manner with the dissolution in the acidic solution. No reaction occurred until the time of $t_{\mathrm{d}}$ or $t_{\mathrm{d}^{\prime}}$ at which the endothermic change was observed. This result means calcium carbonate didn't react in alkaline solution. The CAP film would have dissolved and/or destructed until the time $t_{\mathrm{d}}$ though the heat change due to the CAP dissolution was not detected on the thermogram. Difference between $t_{\mathrm{d}}$ of tablet $\mathbf{A}$ and $t_{\mathrm{d}^{\prime}}$ of tablet $\mathbf{B}$ would be due to the CAP film inhibiting the dissolution of the core tablet. In the time interval between $t_{\mathrm{d}}$ and $t_{\mathrm{e}}$ (or $t_{\mathrm{d}^{\prime}}$ and $t_{\mathrm{e}^{\prime}}$ ), the endothermic change shows that the dissolution of the core 
tablets was occurring. The exothermic change due to the ascorbic acid decomposition was observed after time $t_{\mathrm{e}}$ similarly to that in the acidic solution. In the case of alkaline solution; the neutralization heat of ascorbic acid would exothermically occur. This heat, however; would be produced rapidly in the process of the ascorbic acid dissolution, and so be taken away from the endothermic dissolution heat of the core tablet including ascorbic acid.

TABle II. Characteristic Time in Disintegration Thermogram of Tablet $\mathbf{A}$ and $\mathbf{B}$

\begin{tabular}{|c|c|c|c|c|c|}
\hline \multirow{2}{*}{ Test solution } & \multirow{2}{*}{ Tablet } & \multicolumn{4}{|c|}{ Characteristic time (min) } \\
\hline & & $t_{\mathrm{b}}$ & $t_{\mathrm{c}}$ & $t_{\mathrm{d}}$ & $t_{\theta}$ \\
\hline \multirow[t]{2}{*}{ Acid solution } & $\mathbf{A}$ & 5 & 24 & 38 & 56 \\
\hline & $\mathbf{B}$ & 5 & & $\left.14^{a}\right)$ & 23 \\
\hline \multirow[t]{2}{*}{ Alkaline solution } & $\mathbf{A}$ & 5 & - & 24 & 29 \\
\hline & $\mathbf{B}$ & 5 & 一 & 14 & 20 \\
\hline
\end{tabular}

a) Time $t_{\mathrm{c}}$ and $t_{\mathrm{d}}$ were coincident with each other.

TABLE III. Influence of 18 Months Storage on Dissolution time $t_{\mathrm{e}}$ in the Acidic Solution

\begin{tabular}{lccrrr}
\hline & Tablet & $\begin{array}{c}t_{\mathrm{e}} \\
\text { minutes }\end{array}$ & $\boldsymbol{\sigma}$ & $\boldsymbol{n}$ \\
\hline Before the storage & $\mathbf{A}$ & 56 & 7 & 14 \\
After 18 months & $\mathbf{B}$ & 23 & 3 & 6 \\
& $\mathbf{A}$ & 76 & 37 & 6 \\
& $\mathbf{B}$ & 24 & 4 & 7 \\
\hline
\end{tabular}

Table II lists the characteristic time values of the disintegration obtained from the thermograms of tablet $\mathbf{A}$ and $\mathbf{B}$ in the acidic and alkaline test solution. In the acidic solution, tablet $\mathbf{A}$ showed greater dissolution time $t_{\mathrm{e}}$ than tablet $\mathbf{B}$. This reveals that a protective film inhibited the dissolution of medicinals from the tablet. In the alkaline solution, the difference of $t_{\mathrm{e}}$ between tablet $\mathbf{A}$ and $\mathbf{B}$ was smaller than that in the acidic solution. This result shows the CAP film more easily dissolved in the solution. Table III shows the dissolution time $t_{\mathrm{e}}$ of tablet $\mathbf{A}$ and $\mathbf{B}$ in the acidic solution before and after 18 months storage in well closed container at room temperature. The value of tablet $\mathbf{A}$ increased during the storage in contrast with no change of tablet $\mathbf{B}$. The increase in the dissolution time $t_{\mathrm{e}}$ was 20 minutes for the tablet $\mathbf{A}$ as calculated from Table III. Inhibiting effect of CAP film on dissolution can be quantitatively evaluated from thermogram in term of $\left(t_{\mathrm{d}}-t_{\mathrm{c}}\right)$ of "inhibition times"the time taken for the temperature to be falling at the same rate with the known cooling constant after the exothermic reaction of calcium carbonate. The thermograms of the stored tablet $\mathbf{A}$ gave a value for the inhibition times of 34 minutes. Before the storage, 14 minutes can be calculated for the term from Table II. The difference of the inhibition times before and after the storage was calculated as $34-14=20$ minutes. This value shows the ageing effect of CAP film on the dissolution and is in close agreement with the delay of dissolution of 20 minutes calculated before. Therefore it can be said that the increase in the dissolution time $t_{\mathrm{e}}$ was caused by the chemical and/or physicochemical change of film properties during the storage. Table III also shows that the standard deviation of $t_{\mathrm{e}}$ significantly increased during the storage. The difference of film thickness among the tablet would strongly affect on the dissolution time after the storage since the film became hardly soluble during the period.

A thermogram showd the disintegration and dissolution process of a coated tablet as described above. In the tablet containing many kinds of medicinals, the dissolution heat 
of each medicinals produces together, and each releasing medicinal cannot be separately measured by thermal analysis. However the over all releasing behavior of the medicinals can be estimated from the thermogram. Influence of water protective coating film on dissolution could be clearly reflected on the thermogram, and the different manufacturing procedures of the coating would result in the different thermograms corresponding to the procedures. 